\title{
Effect of Long-Term Antiorthostatic Suspension in a Murine Model of Acute Lung Injury
}

\author{
Tae Young Jang · Ah-Yeoun Jung • Young Hyo Kim \\ Department of Otorhinolaryngology-Head and Neck Surgery, Inha University School of Medicine, Incheon, Korea
}

Objectives. Antiorthostatic suspension (AOS) is ground-based model of simulated microgravity. There is still no study about the effect of long-term microgravity on the clinical course of acute lung injury. We evaluated the effect of simulated microgravity using AOS in a murine model of acute lung injury by lipopolysaccharide (LPS).

Methods. Thirty BALB/c mice were used. During 4 weeks, mice were equally allocated to control (free movement), restraint (tail suspended, but hindlimbs not unloaded), and AOS group (hindlimb unloaded). After then, mice got intranasal challenge with LPS $(20 \mathrm{mg} / \mathrm{kg}, 50 \mu \mathrm{L})$. We measured: weight gain before and after AOS, the number of inflammatory cells and titers of cytokines (interleukin [IL]-1 $\beta$, IL-6, IL-10, tumor necrosis factor- $\alpha$, and interferon- $\gamma$ ) in bronchoalveolar lavage (BAL) fluid, titer of myeloperoxidase (MPO) in serum and lung homogenate, and histopathologic examination of lung tissue.

Results. AOS group had significant weight loss compared to control and restraint group $(P<0.001)$. AOS group also showed significantly decreased lymphocytes $(P=0.023)$ compared to control group. In AOS group, titer for IL- $1 \beta$ in BAL fluid was significantly lower than restraint group $(P=0.049)$. Titer for serum MPO was significantly decreased in AOS group compared to restraint group $(P=0.004)$. However, there was no significant difference of MPO titers in lung tissue between groups. Histopathologic examination of lung tissue revealed no significant difference in the degree of pulmonary infiltration between restraint and AOS group.

Conclusion. In spite of modest anti-inflammatory effect, prolonged AOS caused no significant change in LPS-induced pulmonary inflammation.

Keywords. Hindlimb Suspension; Lipopolysaccharides; Acute Lung Injury

\section{INTRODUCTION}

Antiorthostatic suspension (AOS) is one of well-known ground based model of simulated microgravity [1]. We could perform AOS experiment by simply hanging the tail of mouse so that the hindlimbs are not bearing weight. Using their forelimbs on the ground, animal could move and get food and water [1]. Several researchers have suggested that AOS could reproduce the effect

\footnotetext{
- Received October 20, 2015

Revised November 25, 2015

Accepted December 22, 2015

- Corresponding author: Young Hyo Kim

Department of Otorhinolaryngology-Head and Neck Surgery, Inha

University Hospital, Inha University School of Medicine, 27 Inhang-ro,

Jung-gu, Incheon 22332, Korea

Tel: +82-32-890-2437, Fax: +82-32-890-3580

E-mail: inhaorl@inha.ac.kr
}

of real microgravity on the cardiovascular, musculoskeletal, and more importantly, immune systems of a living organism [2-4].

Acute lung injury is one of common clinical complication of pulmonary infection, and is the cause of respiratory failure [5]. It could be characterized as inflammation of lung parenchyme, including infiltration of neutrophils into the alveoli and increase of several pro-inflammatory cytokines such as tumor necrosis factor (TNF)- $\alpha$, interleukin (IL)-1 $\beta$, and IL-6 [6]. As the era of 'space travel' is near at hand, patients with pulmonary disorders as well as healthy individuals could be exposed to long-term microgravity during space travel. However, there is still no study about the effect of long-term simulated or actual microgravity on the clinical course of acute lung injury.

Therefore, we evaluated the effect of simulated microgravity using AOS in a murine model of acute lung injury by lipopolysaccharide (LPS), by measuring: (1) weight gain before and after

Copyright @ 2016 by Korean Society of Otorhinolaryngology-Head and Neck Surgery.

This is an open-access article distributed under the terms of the Creative Commons Attribution Non-Commercial License (http://creativecommons.org/licenses/by-nc/4.0)

which permits unrestricted non-commercial use, distribution, and reproduction in any medium, provided the original work is properly cited. 
AOS; (2) the number of inflammatory cells and titers of cytokines such as IL-1 $\beta$, IL-6, IL-10, TNF- $\alpha$, and interferon (IFN)- $\gamma$ in bronchoalveolar lavage (BAL) fluid; (3) titer of myeloperoxidase (MPO) in serum and lung homogenate, and finally by (4) histopathologic examination of lung tissue.

\section{MATERIALS AND METHODS}

\section{Animals}

Thirty BALB/c mice, 4 weeks old and free from specific murine pathogens, were used (Orient Bio, Seongnam, Korea). Throughout the whole experiment period, all mice were raised in a specific-pathogen-free environment with regular light-dark cycle and unlimited access to ovalbumin-free water and food. The experimental protocol of our study was approved by the Animal Care and Use Committee of Inha University (INHA 140919327).

\section{Antiorthostatic suspension}

For performing AOS, we suspended a tail of mouse so that only hindlimbs are unloaded, and forelimbs remained weight-bearing (forelimbs on the ground). In order to prevent tail necrosis during AOS, we sealed around tail with sponge and tape, and protected it from being gnawed by placing a plastic tube around the tail. The angle of suspension was about $30^{\circ}$. As we connected a swivel pulley near the tip of tail and suspended it on a steel bar, mice could move freely along the axis of steel bar with free rotation in any direction using their forelimb.

During the period of 4 weeks, mice in control group $(n=10)$ were raised in individual cages without any restraint during the experimental period. Mice in restraint group $(n=10)$ were suspended by their tail, but their hindlimbs were not unloaded (touching the ground). Finally in AOS group $(n=10)$, mice were exposed to continuous AOS.

\section{Induction of acute lung injury}

After 4 weeks, mice in restraint group and AOS group got intranasal instillation of $50 \mu \mathrm{L}$ of $20 \mathrm{mg} / \mathrm{kg}$ lipopolysaccharide (LPS) isolated from Escherichia coli (O111:B4, catalogue \#L4130;

\section{H I G G H L I G G H T S}

- Effect of simulated microgravity was examined in acute lung injury murine model.

- Antiorthostatic suspension (AOS) significantly decreased body weight and lymphocytes.

- AOS also decreased the levels of bronchoalveolar lavage IL-1 $\beta$ and serum myeloperoxidase.

- However, prolonged AOS caused no significant change in histological sections of lung.
Sigma-Aldrich, St. Louis, MO, USA). Mice in control group were given intranasal challenge with $50-\mu \mathrm{L}$ phosphate-buffered saline (PBS) with no LPS. Twenty-four hours after PBS or LPS challenge, all mice were sacrificed.

\section{Weight gain \& differential cell counts in BAL fluid}

Before and after 4 weeks of experimental period, we measured the weight of each mouse. Weight gain was calculated by subtracting initial weight from the final weight after 4 weeks. Immediately after sacrifice, we collected serum by aortic puncture technique. In order to get BAL fluid, we inserted a polyethylene tube into the trachea. Then we performed lavage with sterile saline (about $3 \mathrm{~mL}$ ) through intra-tracheal tube. We stained centrifuged preparations with Diff-Quik (Baxter Scientific, Miami, FL, USA) according to the guideline of manufacturer, and determined differential cell count for inflammatory cells such as eosinophils, neutrophils, lymphocytes and macrophages in high power field $(\times 1,000)$. Two impartial, blinded practitioners counted the number of cells in each slide, and the mean value of them was used.

\section{Cytokines in BAL fluid and myeloperoxidase assay}

We measured the titers of IL-1 $\beta$, IL-6, IL-10, TNF- $\alpha$, and IFN- $\gamma$ in BAL fluid by using appropriate ELISA kits (BioSource International, Camarillo, CA, USA), according to manufacturer's instruction. We also performed MPO assay for serum and lung homogenates using mouse myeloperoxidase DuoSet ELISA kit (R\&D systems, Minneapolis, MN, USA) according to manufacturer's guide.

\section{Histopathologic examination}

Lung tissues were fixed in a $10 \%$ formalin solution for 3 weeks, and then embedded in paraffin. For prepared sections with $4-\mu \mathrm{m}$ thickness, we performed hematoxylin and eosin staining and Sirius red staining. We counted the number of neutrophils infiltrated in a $1 \mathrm{~mm}^{2}$ of lung parenchyme. Again, two blinded practitioners independently counted the number of neutrophils. Then we used the mean value as the number of infiltrated neutrophils.

\section{Statistical analysis}

For the comparison of weight gain, differential cell counts and titers of cytokines in BAL fluid, titers of serum and lung MPO and the number of infiltrated neutrophils into the lung parenchyme, we adopted non-parametric tests such as Kruskal-Wallis test and the Mann-Whitney $U$-test. We expressed all results as means \pm standard deviations. We used IBM SPSS ver. 19.0 (IBM Co., Armonk, NY, USA) and a $P$-value of less than 0.05 was considered statistically significant. 


\section{RESULTS}

\section{Prolonged AOS caused significant weight loss}

After 4 weeks of orthostatic suspension, restraint group had significantly less weight gain compared with control group $(P<0.001)$. After prolonged suspension of 4 weeks, AOS group had remarkable weight loss, with statistical significance compared to control or restraint group $(P<0.001)$ (Fig. 1).

\section{Change of neutrophils, lymphocytes, and macrophages in BAL fluid after prolonged AOS}

The total cell count in BAL fluid was $340.5 \times 10^{3} / \mathrm{mL}$ in control group, $741.4 \times 10^{3} / \mathrm{mL}$ in restraint group, and $601.0 \times 10^{3} / \mathrm{mL}$ in AOS group. Restraint group showed significantly increased neutrophils, slightly decreased lymphocytes, and remarkably decreased macrophages in BAL fluid compared to control group. On the other hand, AOS showed significant increase of neutrophils and decrease of lymphocytes and macrophages, compared with control group $(P<0.001$ for neutrophils and macrophages and $P=0.023$ for lymphocytes). Comparing restraint and AOS group, there was modest tendency of decrease of all inflammatory cells in BAL fluid, although not reaching statistical significance $(P>0.05)$ (Fig. 2).

\section{Significantly decreased IL-1 $\beta$ in response to LPS after prolonged AOS}

After LPS instillation, restraint group showed significant increase of IL-1 $\beta$, IL- 6 , and TNF- $\alpha$ in their BAL fluid, compared to control group. However, after prolonged suspension, AOS group showed significantly lower IL-1 $\beta$ compared with restraint group $(P=0.049)$. Although IL-6 and TNF- $\alpha$ had modest tendency of decrease after AOS, there was no statistical significance $(P>$ 0.05) (Fig. 3).

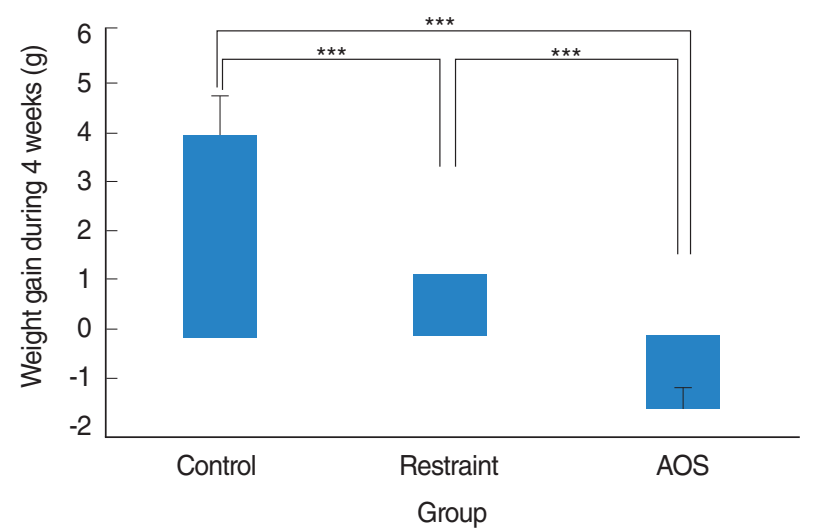

Fig. 1. Weight gain after 4 weeks. Control group: free movement without tail suspension. Restraint group: tail suspended, but hindlimb not unloaded. Antiorthostatic suspension (AOS) group: hindlimb unloaded by tail suspension. Error bar: standard deviation. KruskalWallis and Mann-Whitney U-tests. ${ }^{* \star *} P<0.001$.
AOS caused marked decrease of serum MPO but no decrease of lung MPO

Restraint group showed significant increase of serum MPO titers after LPS instillation, compared to control group $(P<0.001)$. In AOS group, prolonged hindlimb unloading significantly reduced
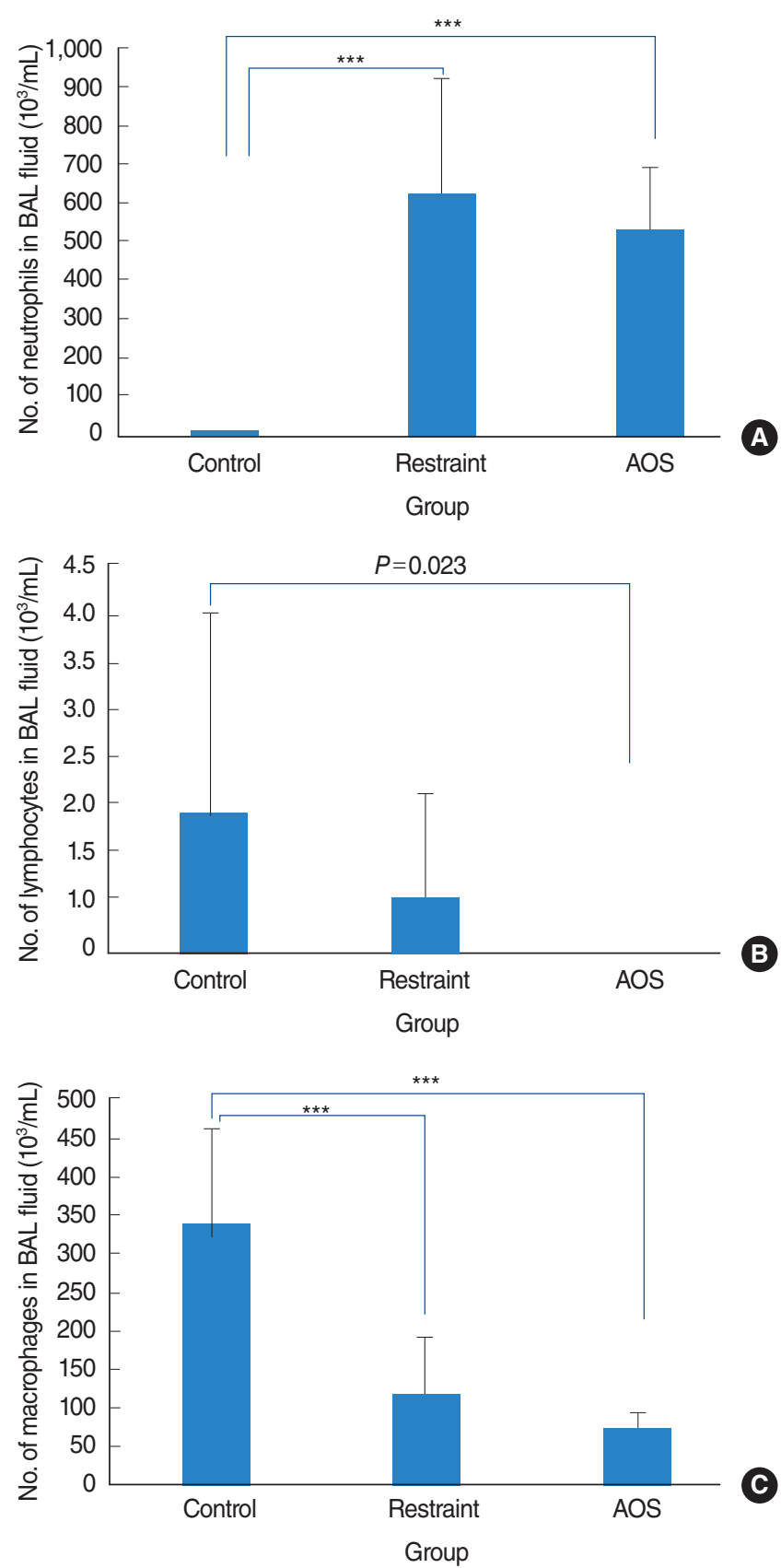

Fig. 2. Number of neutrophils (A), lymphocytes (B), and macrophages $(C)$ in bronchoalveolar lavage (BAL) fluid after intranasal challenge with lipopolysaccharide. Control group: free movement without tail suspension. Restraint group: tail suspended, but hindlimb not unloaded. Antiorthostatic suspension (AOS) group: hindlimb unloaded by tail suspension. Error bar: standard deviation. KruskalWallis and Mann-Whitney $U$-tests. ${ }^{* \star *} P<0.001$. 


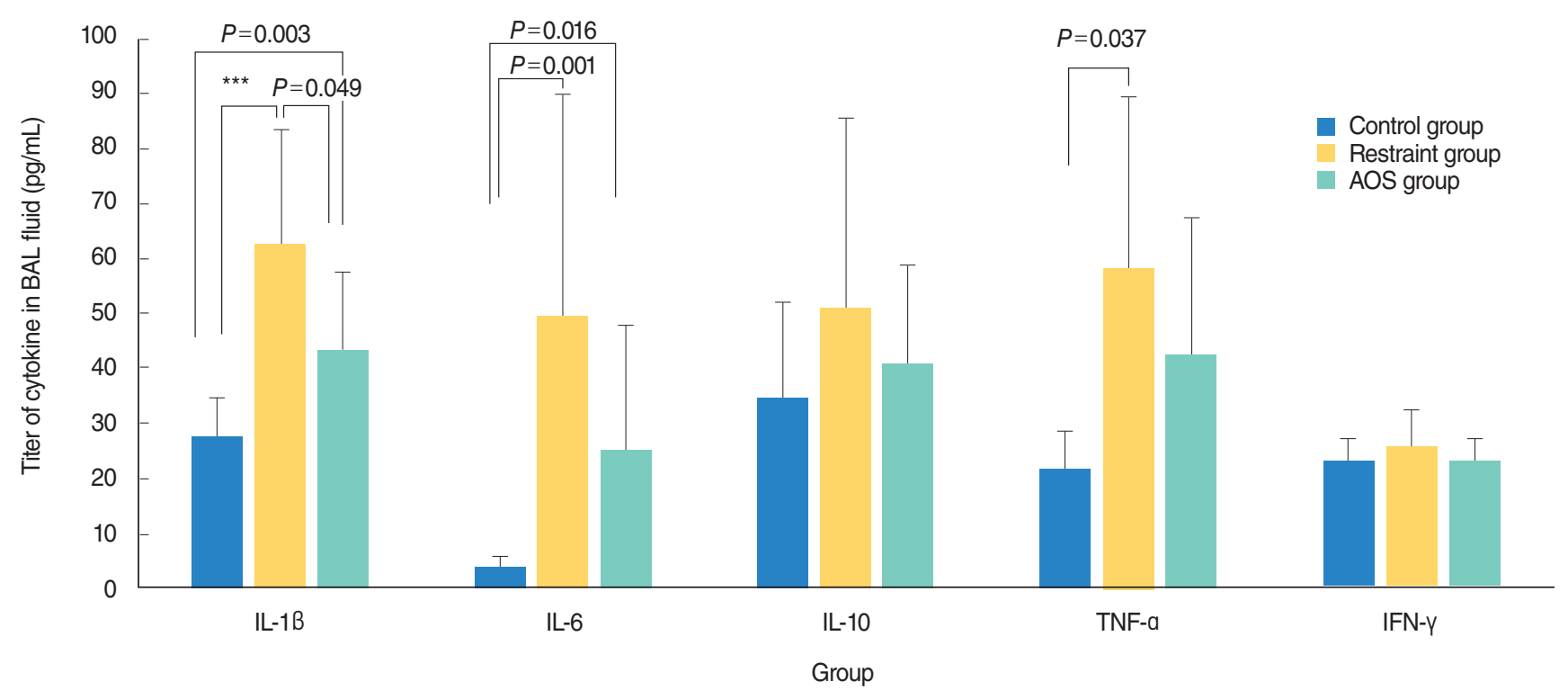

Fig. 3. Titers for interleukin (IL)-1B, IL-6, IL-10, tumor necrosis factor (TNF)-a, and interleukin (IFN)-y in bronchoalveolar lavage (BAL) fluid after intranasal challenge with lipopolysaccharide. Control group: free movement without tail suspension. Restraint group: tail suspended, but hindlimb not unloaded. Antiorthostatic suspension (AOS) group: hindlimb unloaded by tail suspension. Error bar: standard deviation. ${ }^{\star * \star} P<0.001$.
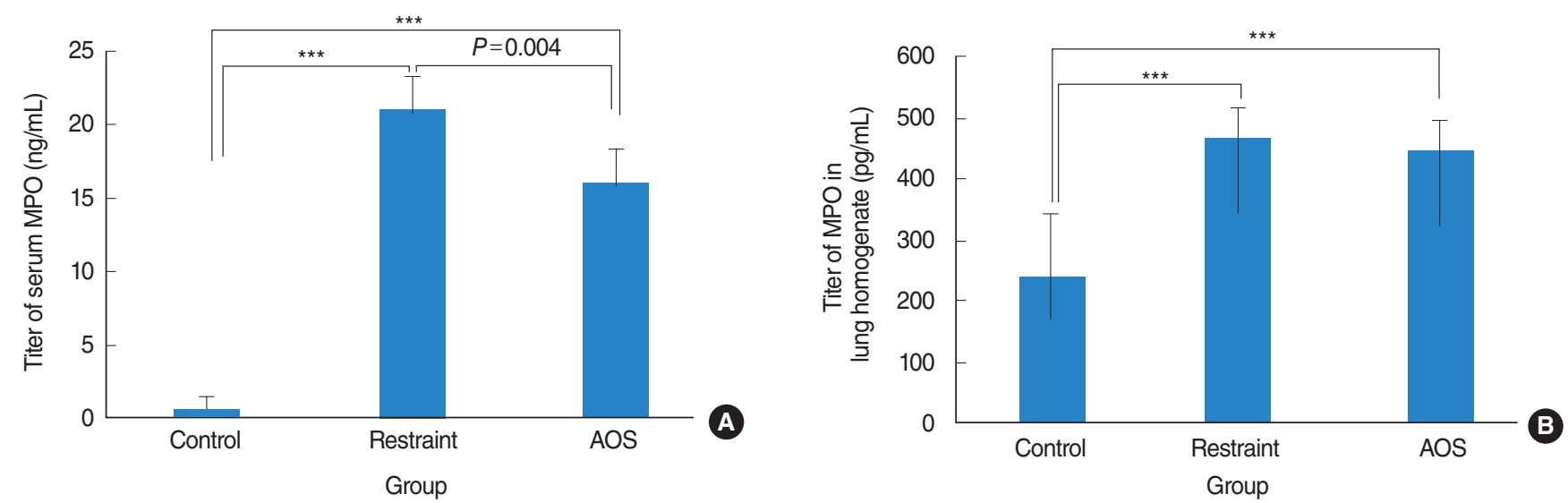

Fig. 4. Titer for serum myeloperoxidase (MPO; A) and MPO titer in lung homogenate (B). Control group: free movement without tail suspension. Restraint group: tail suspended, but hindlimb not unloaded. Antiorthostatic suspension (AOS) group: hindlimb unloaded by tail suspension. Error bar: standard deviation. Kruskal-Wallis and Mann-Whitney U-tests. ${ }^{\star \star *} P<0.001$.

serum MPO titers caused by LPS instillation ( $P=0.004)$ (Fig. $4 \mathrm{~A})$. However, there was no significant difference of MPO in lung homogenates between restraint and AOS group $(P>0.05)$ (Fig. 4B).

\section{Change of neutrophilic infiltration in response to LPS after prolonged AOS}

Compared to control group, restraint and AOS group had significantly more infiltration of inflammatory cells in their pulmonary parenchyme (Fig. 5A). Counting the number of infiltrated neutrophils in $1 \mathrm{~mm}^{2}$ of lung tissue, we could find that there was no significant difference between restraint and AOS group $(P>0.05)$ (Fig. 5B).

\section{DISCUSSION}

In our study, both restraint and AOS groups had significantly less weight gain compared to free control group. It is so remarkable that AOS group had significant weight loss, which was significantly different compared to restraint group. These results well concurs with previous findings. Felix et al. [2] reported significant loss of body weight after 3 weeks of AOS in experimental animals. Lew et al. [7] also found significantly less weight gain in AOS-exposed mice and suggested that the exact pathophysiologic mechanism about the effect of microgravity condition on the energy metabolism had not been solved yet. The possible cause of weight loss in AOS mice could be: (1) reduced food in- 


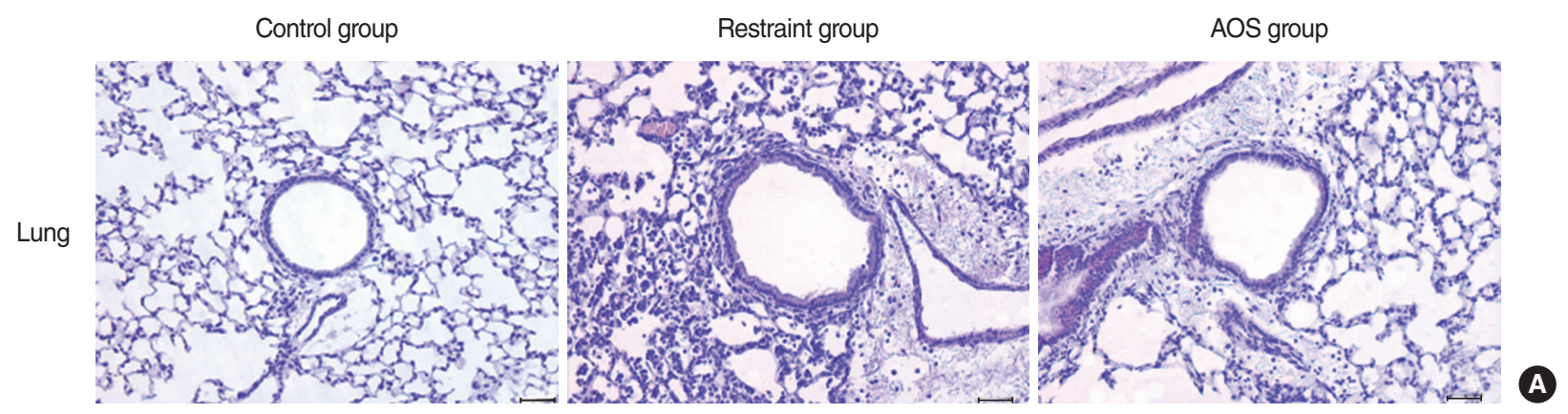

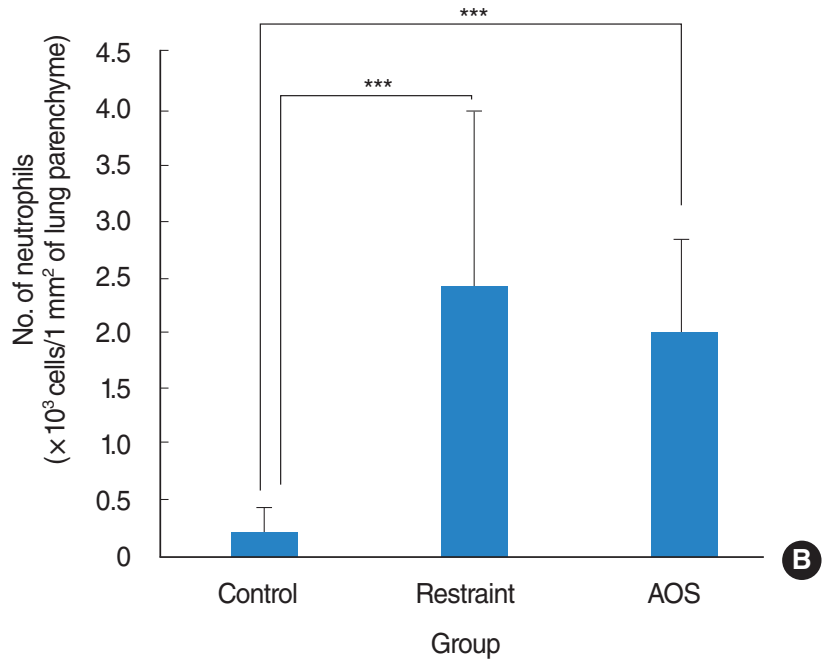

take [8,9], (2) decrease of muscle mass due to disuse atrophy $[10,11]$, and (3) increased energy expenditure [7]. In fact, space travelers suffer from anorexia and weight loss in microgravity condition [12-14]. Therefore, it is essential for him or her to increase food intake and exert muscular exercise to minimize negative energy balance.

There have been some in vitro studies about the effect of simulated or actual microgravity on the function of immune cells. However, to the best of our literature review, we could not find any in vivo study that evaluated different immune cells in BAL fluid. In our study, AOS group had significant decrease of lymphocytes in BAL fluid, which was not in the case in restraint group. It had been known that the blastogenesis of lymphocyte was down-regulated $[15,16]$ and bone marrow cells had less capacities to respond to colony stimulating factors [17]. According to Murdaca et al. [18], when healthy volunteers took a bed rest in antigravity posture for 3 consecutive days, the proliferation of lymphocytes in their serum was significantly decreased. To our best knowledge, our study is the first one to investigate the similar change of lymphocytes in lungs, which is the main target organ in allergic asthma.

Restraint group and AOS group showed significant decrease of macrophages in BAL fluid compared with control group. However, there was no significant difference between restraint and AOS group in the number of macrophages in BAL fluid.
Fig. 5. (A) Histopathologic examination of lung tissue (H\&E, $\times 200$; $50 \mu \mathrm{m}$ ). (B) Number of infiltrated neutrophils in $1 \mathrm{~mm}^{2}$ of lung parenchyme. Control group: free movement without tail suspension. Restraint group: tail suspended, but hindlimb not unloaded. Antiorthostatic suspension (AOS) group: hindlimb unloaded by tail suspension. Error bar: standard deviation. ${ }^{* \star *} P<0.001$.

Macrophages are the major cell population in BAL fluid of healthy animal. However, neutrophils are the predominant cells in BAL fluid. Therefore, the relative proportion of macrophages generally decreases as the proportion of neutrophils increases in BAL fluid. This relative decrease of macrophages in comparison with neutrophils is a natural phenomenon in acute lung injury. However, it does not indicate decreased innate immunity. Miller et al. [19] suggested that the profiles of macrophage activation were increased after 4 days of AOS. However, they also suggested that these results could be highly dependent on several factors, such as the duration of AOS and ability of adaptation in each individual [19]. The duration of AOS in our study was 4 weeks, which is still longer period than previous studies.

Compared to restraint group, mice in AOS group had significantly less IL-1 $\beta$ in BAL fluid when challenged with LPS. According to previous literatures, human monocytic cell line cultured in simulated microgravity secreted significantly less IL-1 $\beta$ in response to phorbol ester challenge $[20,21]$. On the other hand, Wang et al. [22] argued that macrophage cell lines cultured in simulated microgravity had no difference in their secretion of IL-1 $\beta$ compared with those cultured in normal gravity, when they were exposed to LPS challenge. Regarding TNF- $\alpha$, restraint and AOS group showed no difference when exposed to LPS challenge (although some tendency to decrease in AOS group). Hsieh et al. [23] reported that murine macrophage cell 
lines cultured in microgravity condition secreted less TNF- $\alpha$ when challenged with LPS. Our study is, to our best knowledge, the first one that evaluated the effect of simulated microgravity on change of cytokines in response to noxious stimuli in vivo. It could be possible that long term simulated microgravity could have nonspecific inhibitory effect on $\mathrm{T}$ helper (Th) 1 pathway. However, the exact mechanism remains unsolved yet.

There have been some controversies about the change of IL-6 in microgravity. Felix et al. [2] suggested that titer of IL-6 was significantly increased in serum and lymph nodes after 1 week of AOS. According to Lew et al. [7], hypothalamic IL-6 mRNA was up-regulated after AOS. However, Hsieh et al. [23] argued that murine macrophage cell lines cultured in microgravity secreted less IL-6 in response to LPS challenge. Different experimental protocols (in vitro or in vivo, actual or simulated microgravity, duration of exposure, and so on) could cause different results. In our study, there was no significant difference in IL-6 titers between restraint and AOS group. It could be speculated that simulated microgravity had little effect on Th2 pathway in response to toxic stimuli. Also in our study, the titers for IL-10 and IFN- $\gamma$ in BAL fluid was not significantly different between groups.

Neutrophils, recruited by LPS-mediated inflammation, secrete MPO. MPO, in turn, induce production of reactive oxygen species such as superoxide anion [24]. Transcription of mRNA for various peroxide genes were significantly down-regulated in plant during a space flight [25]. In our study, serum MPO in response to LPS challenge was significantly reduced in AOS group, compared to restraint group. Along with decrease of cytokines such as IL-1 $\beta$, decreased serum MPO could be responsible for less pulmonary inflammation.

By histopathologic examination of lung tissue, we could find that there was only marginal difference between restraint and AOS group in the degree of pulmonary inflammation. Although there was less infiltration of neutrophils in the lung parenchyma of AOS group compared to restraint group, that difference did not reach statistical significance. LPS is known to bind the pattern recognition receptor named toll-like receptor 4 and activate intracellular signaling pathway such as nuclear factor-kappa B. Consequently, it activates pro-inflammatory cytokines such as IL-1 $\beta$, promotes recruitment of neutrophils and increases reactive oxygen species [24,26-28]. Although IL-1 $\beta$ in BAL fluid and serum MPO was decreased by prolonged AOS, that was not enough to significantly alleviate pulmonary infiltration significantly. In fact, there was no significant difference comparing MPO of lung homogenate between restraint and AOS group. Miller et al. [19] suggested that AOS for 4 days had protective effect for Listeria monocytogenes infection through activation of macrophages. On the other hand, Belay et al. [29] suggested that mice exposed to AOS for 2 days had less resistance and higher fatality for infection of Klebsiella pneumoniae. In fact, simulated microgravity could have different effect according to target organs. Titers for cytokine in serum could be significantly increased while those in lymph node or in spleen could show absolutely no meaningful change [2]. Further study with more mice, various duration of AOS could yield more meaningful findings in the nearby future.

Simple restraint by tail could induce stress to experimental animal, although they are not antiorthostatically suspended. Therefore, for correct comparison, we set restrain group as orthostaticaly suspended mice (hindlimb touching the ground). By this design, we could exclude the effect of stress induced by restraint itself.

The immune modulatory effect of actual or simulated microgravity could be summarized as: (1) defective and unbalanced immune response due to increase of stress hormone, (2) decrease of delayed hypersensitivity, and (3) decreased resistance to bacterial infection [29]. Furthermore, stress hormone due to the distress during the actual spaceflight or prolonged AOS could cause significant weight loss. Therefore, prolonged microgravity could have a negative effect to an individual during space travel. Further research to prevent these immunologic dysregulation should be performed.

In conclusion, 4 weeks of prolonged AOS induced (1) significant weight loss, (2) significant but borderline decrease of IL-1B, and (3) significant decrease of serum MPO (but not lung MPO). However, it was not conclusive to explain AOS-associated changes in the pulmonary inflammation.

\section{CONFLICT OF INTEREST}

No potential conflict of interest relevant to this article was reported.

\section{ACKNOWLEDGMENTS}

This study was supported by a grant (NRF-2013M1A3A3A 02042309) from the Space Core Technology Development Program of the National Research Foundation (NRF) funded by the Korean Ministry of Science, ICT, and Future Planning (MSIP).

\section{REFERENCES}

1. Gaignier F, Schenten V, De Carvalho Bittencourt M, GauquelinKoch G, Frippiat JP, Legrand-Frossi C. Three weeks of murine hindlimb unloading induces shifts from $\mathrm{B}$ to $\mathrm{T}$ and from th to tc splenic lymphocytes in absence of stress and differentially reduces cell-specific mitogenic responses. PLoS One. 2014 Mar;9(3):e92664.

2. Felix K, Wise K, Manna S, Yamauchi K, Wilson BL, Thomas RL, et al. Altered cytokine expression in tissues of mice subjected to simulated microgravity. Mol Cell Biochem. 2004 Nov;266(1-2):79-85.

3. Morey-Holton E, Globus RK, Kaplansky A, Durnova G. The 
hindlimb unloading rat model: literature overview, technique update and comparison with space flight data. Adv Space Biol Med. 2005 Oct;10:7-40.

4. Sonnenfeld G, Morey ER, Williams JA, Mandel AD. Effect of a simulated weightlessness model on the production of rat interferon. J Interferon Res. 2009 May;2(4):467-70.

5. Bigatello LM, ZapolWM. New approaches to acute lung injury. Br J Anaesth. 1996 Jul;77(1):99-109.

6. Fang L, Gao Y, Liu F, Hou R, Cai RL, Qi Y. Shuang-huang-lian attenuates lipopolysaccharide-induced acute lung injury in mice involving anti-inflammatory and antioxidative activities. Evid Based Complement Alternat Med. 2015;2015:283939.

7. Lew PS, Wong D, Yamaguchi T, Leckstrom A, Schwartz J, Dodd JG, et al.Tail suspension increases energy expenditure independently of the melanocortin system in mice. Can J Physiol Pharmacol. 2009 Oct;87(10):839-49.

8. Amblard D, Lafage-Proust MH, Laib A, Thomas T, Ruegsegger P, Alexandre $\mathrm{C}$, et al. Tail suspension induces bone loss in skeletally mature mice in the $\mathrm{C} 57 \mathrm{BL} / 6 \mathrm{~J}$ strain but not in the $\mathrm{C} 3 \mathrm{H} / \mathrm{HeJ}$ strain. $\mathrm{J}$ Bone Miner Res. 2003 Mar;18(3):561-9.

9. Da Silva MS, Zimmerman PM, Meguid MM, Nandi J, Ohinata K, Xu $\mathrm{Y}$, et al. Anorexia in space and possible etiologies: an overview. Nutrition. 2002 Oct;18(10):805-13.

10. Ferrando AA, Paddon-Jones D, Wolfe RR. Alterations in protein metabolism during space flight and inactivity. Nutrition. 2002 Oct;18 (10):837-41.

11. LeBlanc A, Rowe R, SchneiderV, Evans H, Hedrick T. Regional muscle loss after short duration spaceflight. Aviat Space Environ Med. 1995 Dec;66(12):1151-4.

12. Lane HW, Feeback DL. Water and energy dietary requirements and endocrinology of human space flight. Nutrition. 2002 Oct;18 (10):820-8.

13. Rambaut PC, Leach CS, Leonard JI. Observations in energy balance in man during spaceflight. Am J Physiol. 1977 Nov;233(5):R208-12.

14. Stein TP, Schluter MD. Excretion of IL-6 by astronauts during spaceflight. Am J Physiol. 1994 Mar;266(3 Pt 1):E448-52.

15. Durnova GN, Kaplansky AS, Portugalov VV. Effect of a 22-day space flight on the lymphoid organs of rats. Aviat Space Environ Med. 1976 Jun;47(6):588-91.

16. Taylor GR, Neale LS, Dardano JR. Immunological analyses of U.S. Space Shuttle crewmembers. Aviat Space Environ Med. 1986 Mar; 57(3):213-7.
17. Sonnenfeld G, Mandel AD, Konstantinova IV, Taylor GR, Berry WD, Wellhausen SR, et al. Effects of spaceflight on levels and activity of immune cells. Aviat Space Environ Med. 1990 Jul;61(7):648-53.

18. Murdaca G, Setti M, Brenci S, Fenoglio D, Lantieri P, Indiveri F, et al. Modifications of immunological and neuro-endocrine parameters induced by antiorthostatic bed-rest in human healthy volunteers. Minerva Med. 2003 Dec;94(6):363-78.

19. Miller ES, Bates RA, Koebel DA, Sonnenfeld G. Antiorthostatic suspension stimulates profiles of macrophage activation in mice. Neuroimmunomodulation. 1999 May-Jun;6(3):160-7.

20. Limouse M, Manie S, Konstantinova I, Ferrua B, Schaffar L. Inhibition of phorbol ester-induced cell activation in microgravity. Exp Cell Res. 1991 Nov;197(1):82-6.

21. Schmitt DA, Hatton JP, Emond C, Chaput D, Paris H, Levade T, et al. The distribution of protein kinase $\mathrm{C}$ in human leukocytes is altered in microgravity. FASEB J. 1996 Dec;10(14):1627-34.

22. Wang C, Luo H, Zhu L, Yang F, Chu Z, Tian H, et al. Microgravity inhibition of lipopolysaccharide-induced tumor necrosis factor- $\alpha$ expression in macrophage cells. Inflamm Res. 2014 Jan;63(1):91-8.

23. Hsieh CL, Chao PD, Fang SH. Morin sulphates/glucuronides enhance macrophage function in microgravity culture system. Eur J Clin Invest. 2005 Sep;35(9):591-6.

24. Silva MT. Neutrophils and macrophages work in concert as inducers and effectors of adaptive immunity against extracellular and intracellular microbial pathogens. J Leukoc Biol. 2010 May;87(5):80513.

25. Kwon T, Sparks JA, Nakashima J, Allen SN, Tang Y, Blancaflor EB. Transcriptional response of Arabidopsis seedlings during spaceflight reveals peroxidase and cell wall remodeling genes associated with root hair development. Am J Bot. 2015 Jan;102(1):21-35.

26. Guha M, Mackman N. LPS induction of gene expression in human monocytes. Cell Signal. 2001 Feb;13(2):85-94.

27. Nikaido H. Molecular basis of bacterial outer membrane permeability revisited. Microbiol Mol Biol Rev. 2003 Dec;67(4):593-656.

28. Polumuri SK, Jayakar GG, Shirey KA, Roberts ZJ, Perkins DJ, Pitha PM, et al. Transcriptional regulation of murine IL-33 by TLR and non-TLR agonists. J Immunol. 2012 Jul;189(1):50-60.

29. Belay T, Aviles H,Vance M, Fountain K, Sonnenfeld G. Effects of the hindlimb-unloading model of spaceflight conditions on resistance of mice to infection with Klebsiella pneumoniae. J Allergy Clin Immunol. 2002 Aug;110(2):262-8. 